\title{
SECOND HANKEL DETERMINANT PROBLEM FOR SOME ANALYTIC FUNCTION CLASSES WITH CONNECTED $K$-FIBONACCI NUMBERS
}

\author{
H. ÖZlem Güney, J. SOKÓe, S. İlhaN
}

Abstract. In this paper, we determine upper bound for the second Hankel determinant in some classes of analytic functions in the open unit disc connected with $k$-Fibonacci numbers $F_{k, n}(k>0)$. For this purpose we apply properties of $k$-Fibonacci numbers to consider second Hankel determinant problem for the class $\mathcal{S} \mathcal{L}^{k}$ and $\mathcal{K} \mathcal{S} \mathcal{L}^{k}$. The results presented in this paper have been shown to generalize and improve some recent work of Sokół et al. [18].

2010 Mathematics Subject Classification: Primary 30C45, secondary 30C80.

Keywords: univalent functions, convex functions, starlike functions, subordination, $k$-Fibonacci numbers.

\section{INTRODUCTION}

Let $\mathbb{D}=\{z:|z|<1\}$ be the unit disc in the complex plane. The class of all analytic functions $f(z)=z+\sum_{n=2}^{\infty} a_{n} z^{n}$ in the open unit disc $\mathbb{D}$ with normalization $f(0)=0$, $f^{\prime}(0)=1$ is denoted by $\mathcal{A}$ and the class $\mathcal{S} \subset \mathcal{A}$ is the class which consists of univalent functions in $\mathbb{D}$. We say that $f$ is subordinate to $F$ in $\mathbb{D}$, written as $f \prec F$, if and only if $f(z)=F(\omega(z))$ for some analytic function $\omega,|\omega(z)| \leq|z|, z \in \mathbb{D}$.

Recently, N. Yilmaz Özgür and J. Sokół [12] introduced the class $\mathcal{S} \mathcal{L}^{k}$ of starlike functions connected with $k$-Fibonacci numbers as the set of functions $f \in \mathcal{A}$ which is described in the following definition.

Definition 1. Let $k$ be any positive real number. The function $f \in \mathcal{A}$ belongs to the class $\mathcal{S} \mathcal{L}^{k}$ if it satisfies the condition that

$$
\frac{z f^{\prime}(z)}{f(z)} \prec \widetilde{p}_{k}(z), z \in \mathbb{D},
$$

where

$$
\widetilde{p}_{k}(z)=\frac{1+\tau_{k}^{2} z^{2}}{1-k \tau_{k} z-\tau_{k}^{2} z^{2}}, \tau_{k}=\frac{k-\sqrt{k^{2}+4}}{2}, z \in \mathbb{D} .
$$


H. Özlem Güney, J. Sokół, S. İlhan - Second Hankel determinant problem ...

Now we define the class $\mathcal{K} \mathcal{S} \mathcal{L}^{k}$ as follows:

Definition 2. Let $k$ be any positive real number. The function $f \in \mathcal{A}$ belongs to the class $\mathcal{K} \mathcal{S} \mathcal{L}^{k}$ if it satisfies the condition that

$$
1+\frac{z f^{\prime \prime}(z)}{f^{\prime}(z)} \prec \widetilde{p}_{k}(z), z \in \mathbb{D},
$$

where the function $\widetilde{p}_{k}$ is defined in (2).

For $k=1$, the classes $\mathcal{S} \mathcal{L}^{k}$ and $\mathcal{K} \mathcal{S} \mathcal{L}^{k}$ become the classes $\mathcal{S} \mathcal{L}$ and $\mathcal{K} \mathcal{S} \mathcal{L}$ of shelllike functions defined in [15], see also [16].

It was proved in [12] that functions in the class $\mathcal{S} \mathcal{L}^{k}$ are univalent in $\mathbb{D}$. Moreover, the class $\mathcal{S L}^{k}$ is a subclass of the class of starlike functions $\mathcal{S}^{*}$, even more, starlike of order $k\left(k^{2}+4\right)^{-1 / 2} / 2$. The name attributed to the class $\mathcal{S} \mathcal{L}^{k}$ is motivated by the shape of the curve

$$
\mathcal{C}=\left\{\widetilde{p}_{k}\left(e^{i t}\right): t \in[0,2 \pi) \backslash\{\pi\}\right\} .
$$

The curve $\mathcal{C}$ has a shell-like shape and it is symmetric with respect to the real axis. Its graphic shape, for $k=1$, is given below in Fig.1.

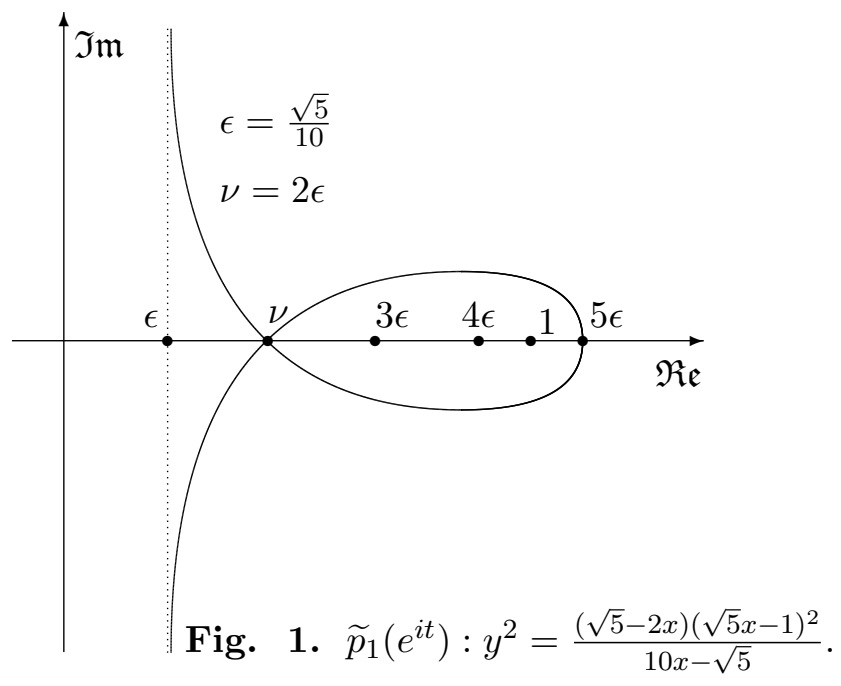

For $k \leq 2$, note that we have

$$
\widetilde{p}_{k}\left(e^{ \pm i \arccos \left(k^{2} / 4\right)}\right)=k\left(k^{2}+4\right)^{-1 / 2}
$$

and so the curve $\mathcal{C}$ intersects itself on the real axis at the point $w_{1}=k\left(k^{2}+4\right)^{-1 / 2}$. Thus $\mathcal{C}$ has a loop intersecting the real axis also at the point $w_{2}=\left(k^{2}+4\right) /(2 k)$. 
H. Özlem Güney, J. Sokół, S. İlhan - Second Hankel determinant problem ...

For $k>2$, the curve $\mathcal{C}$ has no loops and it is like a conchoid, see for details [12]. Moreover, the coefficients of $\widetilde{p}_{k}$ are connected with $k$-Fibonacci numbers.

For any positive real number $k$, the $k$-Fibonacci number sequence $\left\{F_{k, n}\right\}_{n=0}^{\infty}$ is defined recursively by

$$
F_{k, 0}=0, F_{k, 1}=1 \text { and } F_{k, n+1}=k F_{k, n}+F_{k, n-1} \text { for } n \geq 1 .
$$

When $k=1$, we obtain the well-known Fibonacci numbers $F_{n}$. It is known that the $\mathrm{n}^{\text {th }} k$-Fibonacci number is given by

$$
F_{k, n}=\frac{\left(k-\tau_{k}\right)^{n}-\tau_{k}^{n}}{\sqrt{k^{2}+4}},
$$

where $\tau_{k}=\left(k-\sqrt{k^{2}+4}\right) / 2$. If $\widetilde{p}_{k}(z)=1+\sum_{n=1}^{\infty} \widetilde{p}_{k, n} z^{n}$, then we have

$$
\widetilde{p}_{k, n}=\left(F_{k, n-1}+F_{k, n+1}\right) \tau_{k}^{n}, n=1,2,3, \ldots,
$$

see also [12].

Lemma 1. [12] If $f(z)=z+\sum_{n=2}^{\infty} a_{n} z^{n}$ belongs to the class $\mathcal{S} \mathcal{L}^{k}$, then we have

$$
\left|a_{n}\right| \leq\left|\tau_{k}\right|^{n-1} F_{k, n},
$$

where $\tau_{k}=\left(k-\sqrt{k^{2}+4}\right) / 2$. Equality holds in (7) for the function

$$
\begin{aligned}
g_{k}(z) & =\frac{z}{1-k \tau_{k} z-\tau_{k}^{2} z^{2}} \\
& =\sum_{n=1}^{\infty} \tau_{k}^{n-1} F_{k, n} z^{n} \\
& =z+\frac{\left(k-\sqrt{k^{2}+4}\right) k}{2} z^{2}+\left(k^{2}+1\right)\left(\frac{\left(k-\sqrt{k^{2}+4}\right) k}{2}+1\right) z^{3}+\cdots
\end{aligned}
$$

Let $\mathcal{P}(\beta), 0 \leq \beta<1$, denote the class of analytic functions $p$ in $\mathbb{D}$ with $p(0)=1$ and $\operatorname{Re}\{p(z)\}>\beta$. Especially, we use $\mathcal{P}(0)=\mathcal{P}$ as $\beta=0$.

In [12], they proved the following theorem:

Theorem 2. Let $\left\{F_{k, n}\right\}$ be the sequence of $k$-Fibonacci numbers defined in. If

$$
\widetilde{p}_{k}(z)=\frac{1+\tau_{k}^{2} z^{2}}{1-k \tau_{k} z-\tau_{k}^{2} z^{2}}=1+\sum_{n=1}^{\infty} p_{n} z^{n},
$$

where $\tau_{k}=\frac{k-\sqrt{k^{2}+4}}{2}, z \in \mathbb{D}$, then we have

$$
p_{n}=\left(F_{k, n-1}+F_{k, n+1}\right) \tau_{k}^{n}, \quad n=1,2,3, \ldots
$$


H. Özlem Güney, J. Sokół, S. İlhan - Second Hankel determinant problem ...

We will use the following lemma for proving our main result.

Lemma 3. [13] Let $p \in \mathcal{P}$ with $p(z)=1+c_{1} z+c_{2} z^{2}+\cdots$, then

$$
\left|c_{n}\right| \leq 2, \quad \text { for } \quad n \geq 1 \text {. }
$$

If $\left|c_{1}\right|=2$, then $p(z) \equiv p_{1}(z) \equiv(1+x z) /(1-x z)$ with $x=\frac{c_{1}}{2}$. Conversely, if $p(z) \equiv p_{1}(z)$ for some $|x|=1$, then $c_{1}=2 x$. Furthermore, we have

$$
\left|c_{2}-\frac{c_{1}^{2}}{2}\right| \leq 2-\frac{\left|c_{1}\right|^{2}}{2} .
$$

If $\left|c_{1}\right|<2$, and $\left|c_{2}-\frac{c_{1}^{2}}{2}\right|=2-\frac{\left|c_{1}\right|^{2}}{2}$, then $p(z) \equiv p_{2}(z)$, where

$$
p_{2}(z)=\frac{1+\bar{x} w z+z(w z+x)}{1+\bar{x} w z-z(w z+x)}
$$

and $x=\frac{c_{1}}{2}, w=\frac{2 c_{2}-c_{1}^{2}}{4-\left|c_{1}\right|^{2}}$ and $\left|c_{2}-\frac{c_{1}^{2}}{2}\right|=2-\frac{\left|c_{1}\right|^{2}}{2}$.

Lemma 4. ([9]) Let $p \in \mathcal{P}$ with coefficients $c_{n}$ as above, then

$$
\left|c_{3}-2 c_{1} c_{2}+c_{1}^{3}\right| \leq 2
$$

In 1976, Noonan and Thomas [10] stated the $s^{\text {th }}$ Hankel determinant for $s \geq 1$ and $q \geq 1$ as

$$
H_{s}(q)=\left|\begin{array}{cccc}
a_{q} & a_{q+1} & \ldots & a_{q+s-1} \\
a_{q+1} & a_{q+2} & \ldots & \vdots \\
\vdots & \vdots & \vdots & \vdots \\
a_{q+s-1} & \ldots & \ldots & a_{q+2(s-1)}
\end{array}\right|
$$

where $a_{1}=1$.

This determinant has also been considered by several authors. For example, Noor [11] determined the rate of growth of $H_{s}(q)$ as $q \rightarrow \infty$ for functions $f$ in $\mathcal{S}$ with bounded boundary. Ehrenborg in [3] studied the Hankel determinant of exponential polynomials. The Hankel transform of an integer sequence and some of its properties were discussed by Layman in [8]. Also, several authors considered the case $s=2$. Especially, $H_{2}(1)=a_{3}-a_{2}^{2}$ is known as Fekete-Szegö functional and this functional is generalized to $a_{3}-\mu a_{2}^{2}$ where $\mu$ is some real number [4]. Estimating for an upper bound of $\left|H_{2}(1)\right|$ is known as the Fekete-Szegö problem. Raina and Sokół considered Fekete-Szegö problem for the class $\mathcal{S} \mathcal{L}$ in [14] and for the class $\mathcal{S} \mathcal{L}^{k}$ in [17]. In 1969, Keogh and Merkes [7] solved this problem for the classes $\mathcal{S}^{*}$ and $\mathcal{C}$. 
H. Özlem Güney, J. Sokół, S. İlhan - Second Hankel determinant problem ...

The second Hankel determinant is $H_{2}(2)=a_{2} a_{4}-a_{3}^{2}$. Janteng [5] found the sharp upper bound for $\left|H_{2}(2)\right|$ for univalent functions whose derivative has positive real part. In [6] Janteng et al. obtained the bounds for $\left|H_{2}(2)\right|$ for the classes $\mathcal{S}^{*}$ and $\mathcal{C}$. Also, Sokół et al. considered second Hankel determinant problem for the classes $\mathcal{S} \mathcal{L}$ and $\mathcal{K} \mathcal{S} \mathcal{L}$ in [18].

\section{The Second Hankel Determinant Problem}

Let we prove the coefficient bound of the function in the class $\mathcal{K} \mathcal{S} \mathcal{L}^{k}$ as follows:

Theorem 5. If $f(z)=z+\sum_{n=2}^{\infty} a_{n} z^{n}$ belongs to the class $\mathcal{K} \mathcal{S} \mathcal{L}^{k}$, then we have

$$
\left|a_{n}\right| \leq \frac{\left|\tau_{k}\right|^{n-1} F_{k, n}}{n}
$$

where $\tau_{k}=\left(k-\sqrt{k^{2}+4}\right) / 2$. Equality holds in (7) for the function

$$
f_{k}(z)=\frac{1}{1+\tau_{k}^{2}} \log \frac{1+z}{1-\tau_{k}^{2} z} .
$$

Proof. A function $f$ is in the class $\mathcal{K} \mathcal{S} \mathcal{L}^{k}$ if and only if the function

$$
g(z)=z f^{\prime}(z)
$$

is in the class $\mathcal{S L}^{k}$. The relations (17) follows (3). Therefore, if

$$
z f^{\prime}(z)=z+\sum_{n=2}^{\infty} n a_{n} z^{n} \quad(z \in \mathbb{D})
$$

belongs to the class $\mathcal{S} \mathcal{L}^{k}$, then from Lemma (1), we can write $\left|n a_{n}\right| \leq\left|\tau_{k}\right|^{n-1} F_{k, n}$, which implies (15). The equation (16) is such that $z f_{k}^{\prime}(z)=g_{k}(z)$ where the function $g_{k}$ is given in (8), and so from (17), it follows that $f_{k} \in \mathcal{K} \mathcal{S} \mathcal{L}^{k}$. Also, by (8) we have

$$
f_{k}(z)=z+\sum_{n=2}^{\infty} \frac{\left|\tau_{k}\right|^{n-1} F_{k, n}}{n} z^{n} \quad(z \in \mathbb{D}) .
$$

Consequently, the result (7) is sharp.

In [17], Sokol et. al proved the following coefficient bounds: 
H. Özlem Güney, J. Sokół, S. İlhan - Second Hankel determinant problem ...

Theorem 6. If $p(z)=1+p_{1} z+p_{2} z^{2}+\cdots$ and

$$
p(z) \prec \widetilde{p}_{k}(z)=\frac{1+\tau_{k}^{2} z^{2}}{1-k \tau_{k} z-\tau_{k}^{2} z^{2}}, \tau_{k}=\frac{k-\sqrt{k^{2}+4}}{2}, z \in \mathbb{D},
$$

then we have

$$
\left|p_{1}\right| \leq \frac{\left(\sqrt{k^{2}+4}-k\right) k}{2}
$$

and

$$
\left|p_{2}\right| \leq\left(k^{2}+2\right)\left\{\frac{\left(k-\sqrt{k^{2}+4}\right) k}{2}+1\right\} .
$$

The above estimations are sharp.

Now, our first main result (Theorem 7 below) gives an upper bound for the coefficient $p_{3}$.

Theorem 7. If $p(z)=1+p_{1} z+p_{2} z^{2}+\cdots$ and

$$
p(z) \prec \widetilde{p}_{k}(z)=\frac{1+\tau_{k}^{2} z^{2}}{1-k \tau_{k} z-\tau_{k}^{2} z^{2}}, \tau_{k}=\frac{k-\sqrt{k^{2}+4}}{2}, z \in \mathbb{D},
$$

then we have

$$
\left|p_{3}\right| \leq\left(k^{3}+3 k\right)\left\{\frac{\sqrt{k^{2}+4}-k}{2}\right\}^{3} .
$$

The above estimation is sharp.

Proof. If $p \prec \widetilde{p}_{k}$, then there exists an analytic function $w$ such that $|w(z)| \leq|z|$ in $\mathbb{D}$ and $p(z)=\widetilde{p}_{k}(w(z))$. Therefore, the function

$$
h(z)=\frac{1+w(z)}{1-w(z)}=1+c_{1} z+c_{2} z+\cdots \quad(z \in \mathbb{D})
$$

is in the class $\mathcal{P}$. It follows that

$$
w(z)=\frac{c_{1} z}{2}+\left(c_{2}-\frac{c_{1}^{2}}{2}\right) \frac{z^{2}}{2}+\cdots
$$


H. Özlem Güney, J. Sokół, S. İlhan - Second Hankel determinant problem ...

and

$$
\begin{aligned}
\widetilde{p}_{k}(w(z)) & =1+\widetilde{p}_{k, 1}\left\{\frac{c_{1} z}{2}+\left(c_{2}-\frac{c_{1}^{2}}{2}\right) \frac{z^{2}}{2}+\cdots\right\}+\widetilde{p}_{k, 2}\left\{\frac{c_{1} z}{2}+\left(c_{2}-\frac{c_{1}^{2}}{2}\right) \frac{z^{2}}{2}+\cdots\right\}^{2} \\
& +\widetilde{p}_{k, 3}\left\{\frac{c_{1} z}{2}+\left(c_{2}-\frac{c_{1}^{2}}{2}\right) \frac{z^{2}}{2}+\left(c_{3}-c_{1} c_{2}+\frac{c_{1}^{3}}{4}\right) \frac{z^{3}}{2}+\cdots\right\}^{3} \cdots \\
& =1+\frac{\widetilde{p}_{k, 1} c_{1}}{2} z+\left\{\frac{1}{2}\left(c_{2}-\frac{c_{1}^{2}}{2}\right) \widetilde{p}_{k, 1}+\frac{1}{4} c_{1}^{2} \widetilde{p}_{k, 2}\right\} z^{2} \\
& +\left\{\frac{1}{2}\left(c_{3}-c_{1} c_{2}+\frac{c_{1}^{3}}{4}\right) \tilde{p}_{k, 1}+\frac{1}{2} c_{1}\left(c_{2}-\frac{c_{1}^{2}}{2}\right) \widetilde{p}_{k, 2}+\frac{c_{1}^{3}}{8} \widetilde{p}_{k, 3}\right\} z^{3}+\cdots \\
& =p(z) .
\end{aligned}
$$

From (6), we find the coefficients $\widetilde{p}_{k, n}$ of the function $\widetilde{p}_{k}$ given by

$$
\widetilde{p}_{k, n}=\left(F_{k, n-1}+F_{k, n+1}\right) \tau_{k}^{n} .
$$

This shows the relevant connection $\widetilde{p}_{k}$ with the sequence of $k$-Fibonacci numbers

$$
\begin{aligned}
\widetilde{p}_{k}(z) & =1+\sum_{n=1}^{\infty} \widetilde{p}_{k, n} z^{n} \\
& =1+\left(F_{k, 0}+F_{k, 2}\right) \tau_{k} z+\left(F_{k, 1}+F_{k, 3}\right) \tau_{k}^{2} z^{2}+\cdots \\
& =1+k \tau_{k} z+\left(k^{2}+2\right) \tau_{k}^{2} z^{2}+\left(k^{3}+3 k\right) \tau_{k}^{3} z^{3}+\cdots
\end{aligned}
$$

If $p(z)=1+p_{1} z+p_{2} z^{2}+\cdots$, then by (24) and (25), we have

$$
\begin{gathered}
p_{1}=\frac{k \tau_{k} c_{1}}{2} \\
p_{2}=\frac{k \tau_{k}}{2}\left(c_{2}-\frac{c_{1}^{2}}{2}\right)+\frac{\left(k^{2}+2\right)}{4} c_{1}^{2} \tau_{k}^{2}
\end{gathered}
$$

and

$$
p_{3}=\frac{k \tau_{k}}{2}\left(c_{3}-c_{1} c_{2}+\frac{c_{1}^{3}}{4}\right)+\frac{\left(k^{2}+2\right)}{2} c_{1}\left(c_{2}-\frac{c_{1}^{2}}{2}\right) \tau_{k}^{2}+\frac{\left(k^{3}+3 k\right)}{8} c_{1}^{3} \tau_{k}^{3} .
$$

We know that

$$
\tau_{k}\left(k-\tau_{k}\right)=-1,
$$


H. Özlem Güney, J. Sokół, S. İlhan - Second Hankel determinant problem ...

where $\tau_{k}=\frac{k-\sqrt{k^{2}+4}}{2}$.

Now taking absolute value of (28) and using (29), we can write

$$
\begin{aligned}
\left|p_{3}\right| & =\left|\frac{k \tau_{k}}{2}\left(c_{3}-c_{1} c_{2}+\frac{c_{1}^{3}}{4}\right)+\frac{\left(k^{2}+2\right)}{2} c_{1}\left(c_{2}-\frac{c_{1}^{2}}{2}\right) \tau_{k}^{2}+\frac{\left(k^{3}+3 k\right)}{8} c_{1}^{3} \tau_{k}^{3}\right| \\
& =\left|\frac{k \tau_{k}}{2}\left(c_{3}-c_{1} c_{2}+\frac{c_{1}^{3}}{4}\right)+\frac{\left(k^{2}+2\right)}{2} c_{1}\left(c_{2}-\frac{c_{1}^{2}}{2}\right)\left(k \tau_{k}+1\right)+\frac{\left(k^{3}+3 k\right)}{8} c_{1}^{3}\left(\left(k^{2}+1\right) \tau_{k}+k\right)\right| \\
& =\mid\left\{\frac{1}{2}\left(c_{3}-2 c_{1} c_{2}+c_{1}^{3}\right) k-\frac{\left(k^{5}+2 k^{3}-4 k\right)}{4} c_{1}\left(c_{2}-\frac{c_{1}^{2}}{2}\right)+\frac{\left(k^{5}+4 k^{3}+2 k\right)}{4} c_{1} c_{2}\right\} \tau_{k} \\
& +\left\{\frac{\left(k^{2}+2\right)}{2} c_{1}\left(c_{2}-\frac{c_{1}^{2}}{2}\right)+\frac{k\left(k^{3}+3 k\right)}{8} c_{1}^{3}\right\} \mid .
\end{aligned}
$$

From (2) and (5), we find that

$$
\forall n \in \mathbb{N}, \tau_{k}=\frac{\tau_{k}^{n}}{F_{k, n}}-x_{k, n}, \quad x_{k, n}=\frac{F_{k, n-1}}{F_{k, n}}, \quad \lim _{n \rightarrow \infty} \frac{F_{k, n-1}}{F_{k, n}}=\left|\tau_{k}\right| .
$$

Therefore, we have

$$
\begin{aligned}
\left|p_{3}\right|= & \mid\left\{\frac{1}{2}\left(c_{3}-2 c_{1} c_{2}+c_{1}^{3}\right) k-\frac{\left(k^{5}+2 k^{3}-4 k\right)}{4} c_{1}\left(c_{2}-\frac{c_{1}^{2}}{2}\right)+\frac{\left(k^{5}+4 k^{3}+2 k\right)}{4} c_{1} c_{2}\right\} \frac{\tau_{k}^{n}}{F_{k, n}} \\
& +\left\{-\frac{1}{2}\left(c_{3}-2 c_{1} c_{2}+c_{1}^{3}\right) k x_{k, n}+\frac{\left(-k^{4}-k^{2}+4\right)+\left(k^{5}+2 k^{3}-4 k\right) x_{k, n}}{4} c_{1}\left(c_{2}-\frac{c_{1}^{2}}{2}\right)\right. \\
& \left.+\frac{k\left(k^{3}+3 k\right)-\left(k^{5}+4 k^{3}+2 k\right) x_{k, n}}{4} c_{1} c_{2}\right\} \mid \\
\leq & \left|\left\{\frac{1}{2}\left(c_{3}-2 c_{1} c_{2}+c_{1}^{3}\right) k-\frac{\left(k^{5}+2 k^{3}-4 k\right)}{4} c_{1}\left(c_{2}-\frac{c_{1}^{2}}{2}\right)+\frac{\left(k^{5}+4 k^{3}+2 k\right)}{4} c_{1} c_{2}\right\}\right| \mid \frac{\left|\tau_{k}\right|^{n}}{F_{k, n}} \\
& +\mid-\frac{1}{2}\left(c_{3}-2 c_{1} c_{2}+c_{1}^{3}\right) k x_{k, n}+\frac{\left(-k^{4}-k^{2}+4\right)+\left(k^{5}+2 k^{3}-4 k\right) x_{k, n}}{4} c_{1}\left(c_{2}-\frac{c_{1}^{2}}{2}\right) \\
& +\frac{k\left(k^{3}+3 k\right)-\left(k^{5}+4 k^{3}+2 k\right) x_{k, n}}{4} c_{1} c_{2} \mid \\
\leq & \left|\left\{\frac{1}{2}\left(c_{3}-2 c_{1} c_{2}+c_{1}^{3}\right) k-\frac{\left(k^{5}+2 k^{3}-4 k\right)}{4} c_{1}\left(c_{2}-\frac{c_{1}^{2}}{2}\right)+\frac{\left(k^{5}+4 k^{3}+2 k\right)}{4} c_{1} c_{2}\right\}\right| \mid \frac{\left|\tau_{k}\right|^{n}}{F_{k, n}} \\
& +\frac{k}{2}\left|c_{3}-2 c_{1} c_{2}+c_{1}^{3}\right| x_{k, n}+\frac{\left|\left(-k^{4}-k^{2}+4\right)+\left(k^{5}+2 k^{3}-4 k\right) x_{k, n}\right|}{4}\left|c_{1}\right|\left|c_{2}-\frac{c_{1}^{2}}{2}\right| \\
& +\frac{\left|k\left(k^{3}+3 k\right)-\left(k^{5}+4 k^{3}+2 k\right) x_{k, n}\right|}{4}\left|c_{1}\right|\left|c_{2}\right|
\end{aligned}
$$

By (30), for sufficiently large $n$ we have

$$
\forall k,\left|k\left(k^{3}+3 k\right)-\left(k^{5}+4 k^{3}+2 k\right) x_{k, n}\right|=\left(k^{5}+4 k^{3}+2 k\right) x_{k, n}-k\left(k^{3}+3 k\right)
$$

and

$\forall k,\left|\left(-k^{4}-k^{2}+4\right)+\left(k^{5}+2 k^{3}-4 k\right) x_{k, n}\right|=\left(-k^{4}-k^{2}+4\right)+\left(k^{5}+2 k^{3}-4 k\right) x_{k, n}$. 
H. Özlem Güney, J. Sokół, S. İlhan - Second Hankel determinant problem ...

Therefore, from (11), (12) and (13) we can write for sufficiently large $n$

$$
\begin{aligned}
\left|p_{3}\right| \leq & \left\{\left\{\frac{1}{2}\left(c_{3}-2 c_{1} c_{2}+c_{1}^{3}\right) k-\frac{\left(k^{5}+2 k^{3}-4 k\right)}{4} c_{1}\left(c_{2}-\frac{c_{1}^{2}}{2}\right)+\frac{\left(k^{5}+4 k^{3}+2 k\right)}{4} c_{1} c_{2}\right\} \mid \frac{\left|\tau_{k}\right|^{n}}{F_{k, n}}\right. \\
& +\left\{k x_{k, n}+\frac{\left|\left(-k^{4}-k^{2}+4\right)+\left(k^{5}+2 k^{3}-4 k\right) x_{k, n}\right|+\left|k\left(k^{3}+3 k\right)-\left(k^{5}+4 k^{3}+2 k\right) x_{k, n}\right|}{2}\left|c_{1}\right|\right. \\
& \left.-\frac{\left|\left(-k^{4}-k^{2}+4\right)+\left(k^{5}+2 k^{3}-4 k\right) x_{k, n}\right|}{8}\left|c_{1}\right|^{3}\right\} \\
= & \left|\left\{\frac{1}{2}\left(c_{3}-2 c_{1} c_{2}+c_{1}^{3}\right) k-\frac{\left(k^{5}+2 k^{3}-4 k\right)}{4} c_{1}\left(c_{2}-\frac{c_{1}^{2}}{2}\right)+\frac{\left(k^{5}+4 k^{3}+2 k\right)}{4} c_{1} c_{2}\right\}\right| \frac{\left|\tau_{k}\right|^{n}}{F_{k, n}} \\
& +\left\{k x_{k, n}+\frac{\left(-k^{4}-k^{2}+4\right)+\left(k^{5}+2 k^{3}-4 k\right) x_{k, n}+\left(k^{5}+4 k^{3}+2 k\right) x_{k, n}-k\left(k^{3}+3 k\right)}{2}\left|c_{1}\right|\right. \\
& \left.-\frac{\left(-k^{4}-k^{2}+4\right)+\left(k^{5}+2 k^{3}-4 k\right) x_{k, n}}{8}\left|c_{1}\right|^{3}\right\} \\
= & \left|\left\{\frac{1}{2}\left(c_{3}-2 c_{1} c_{2}+c_{1}^{3}\right) k-\frac{\left(k^{5}+2 k^{3}-4 k\right)}{4} c_{1}\left(c_{2}-\frac{c_{1}^{2}}{2}\right)+\frac{\left(k^{5}+4 k^{3}+2 k\right)}{4} c_{1} c_{2}\right\}\right| \frac{\left|\tau_{k}\right|^{n}}{F_{k, n}} \\
& +\left\{k x_{k, n}+\frac{\left(-2 k^{4}-4 k^{2}+4\right)+\left(2 k^{5}+6 k^{3}-2 k\right) x_{k, n}}{2}\left|c_{1}\right|\right. \\
& \left.-\frac{\left(-k^{4}-k^{2}+4\right)+\left(k^{5}+2 k^{3}-4 k\right) x_{k, n}}{8}\left|c_{1}\right|^{3}\right\} .
\end{aligned}
$$

Denote

$$
\begin{aligned}
\left|c_{1}\right|=y, \quad f(y)= & \left\{k x_{k, n}+\frac{\left(-2 k^{4}-4 k^{2}+4\right)+\left(2 k^{5}+6 k^{3}-2 k\right) x_{k, n}}{2} y\right. \\
& \left.-\frac{\left(-k^{4}-k^{2}+4\right)+\left(k^{5}+2 k^{3}-4 k\right) x_{k, n}}{8} y^{3}\right\}, \quad y \in[0,2] .
\end{aligned}
$$

It is easy to check that $f^{\prime}(y)>0$ for $y \in[0,2]$ and for sufficiently large $n$. Since then, for sufficiently large $n$, we have

$$
\max _{y \in[0,2]}\{f(y)\}=\left(k^{5}+4 k^{3}+3 k\right) x_{k, n}-k\left(k^{3}+3 k\right) \text { at } y=2 .
$$

Therefore, we have

$$
\begin{aligned}
\lim _{n \rightarrow \infty} \max _{y \in[0,2]}\{f(y)\} & =\left(k^{5}+4 k^{3}+3 k\right)\left|\tau_{k}\right|-k\left(k^{3}+3 k\right) \\
& =\left(k^{2}+1\right)\left(k^{3}+3 k\right)\left|\tau_{k}\right|-k\left(k^{3}+3 k\right) \\
& =\left(\left(k^{2}+1\right)\left|\tau_{k}\right|-k\right)\left(k^{3}+3 k\right) \\
& =\left(k^{3}+3 k\right)\left|\tau_{k}\right|^{3} .
\end{aligned}
$$


H. Özlem Güney, J. Sokół, S. İlhan - Second Hankel determinant problem ...

Hence, we get

$$
\begin{aligned}
& \lim _{n \rightarrow \infty}\left[\left\{k+\frac{\left|k^{5}+2 k^{3}-4 k\right|+k^{5}+4 k^{3}+2 k}{2}\left|c_{1}\right|-\frac{\left|k^{5}+2 k^{3}-4 k\right|}{8}\left|c_{1}\right|^{3}\right\} \frac{\left|\tau_{k}\right|^{n}}{F_{k, n}}\right. \\
& +\left\{k x_{k, n}+\frac{\left|\left(-k^{4}-k^{2}+4\right)+\left(k^{5}+2 k^{3}-4 k\right) x_{k, n}\right|+\left|k\left(k^{3}+3 k\right)-\left(k^{5}+4 k^{3}+2 k\right) x_{k, n}\right|}{2}\left|c_{1}\right|\right. \\
& \left.\left.-\frac{\left|\left(-k^{4}-k^{2}+4\right)+\left(k^{5}+2 k^{3}-4 k\right) x_{k, n}\right|}{8}\left|c_{1}\right|^{3}\right\}\right] \\
& =\left(k^{3}+3 k\right)\left|\tau_{k}\right|^{3} \\
& =\left(k^{3}+3 k\right)\left\{\frac{\sqrt{k^{2}+4}-k}{2}\right\}^{3}
\end{aligned}
$$

which shows that

$$
\left|p_{3}\right| \leq\left(k^{3}+3 k\right)\left\{\frac{\sqrt{k^{2}+4}-k}{2}\right\}^{3} .
$$

If we take

$$
h(z)=\frac{1+z}{1-z}=1+2 z+2 z^{2}+\ldots,
$$

then putting $c_{1}=c_{2}=c_{3}=2$ in $(28)$ gives $p_{3}=\left(k^{3}+3 k\right)\left\{\frac{\sqrt{k^{2}+4}-k}{2}\right\}^{3}$ and it shows that (22) is sharp. It completes the proof.

Conjecture. If $p(z)=1+p_{1} z+p_{2} z^{2}+\cdots$, and $p \prec \tilde{p}$, then

$$
\left|p_{n}\right| \leq\left(F_{k, n-1}+F_{k, n+1}\right)\left|\tau_{k}\right|^{n}, \quad n=1,2,3, \ldots,
$$

where $F_{k, 0}=0, F_{k, 1}=1$ and $F_{k, n+1}=k F_{k, n}+F_{k, n-1}$ for $n \geq 1$ is the $k$-Fibonacci sequence. This bound would be sharp for the function (25).

This conjecture has been just verified for $n=3$ in last Theorem (7), while for $n=1,2$ it was proved in [17].

Theorem 8. If $f(z)=z+a_{2} z^{2}+\ldots$ belongs to $\mathcal{S} \mathcal{L}^{k}$, then

$$
\left|a_{2} a_{4}-a_{3}^{2}\right| \leq \frac{2 k^{4}+6 k^{2}+3}{3}\left\{\frac{\sqrt{k^{2}+4}-k}{2}\right\}^{4} .
$$

Proof. For given $f \in \mathcal{S} \mathcal{L}^{k}$, define $p(z)=1+p_{1} z+p_{2}^{2} z^{2}+\cdots$, by

$$
\frac{z f^{\prime}(z)}{f(z)}=p(z)
$$


H. Özlem Güney, J. Sokół, S. İlhan - Second Hankel determinant problem ...

where $p \prec \tilde{p}$. Hence

$\frac{z f^{\prime}(z)}{f(z)}=1+a_{2} z+\left(2 a_{3}-a_{2}^{2}\right) z^{2}+\left(3 a_{4}-3 a_{2} a_{3}+a_{2}^{3}\right) z^{3}+\cdots=1+p_{1} z+p_{2}^{2} z^{2}+\cdots$

and

$$
a_{2}=p_{1}, \quad a_{3}=\frac{p_{1}^{2}+p_{2}}{2}, \quad a_{4}=\frac{p_{1}^{3}+3 p_{1} p_{2}+2 p_{3}}{6} .
$$

Therefore,

$$
a_{2} a_{4}-a_{3}^{2}=\frac{1}{12}\left(-p_{1}^{4}+4 p_{1} p_{3}-3 p_{2}^{2}\right)
$$

Using Theorem (6) and Theorem (7), we obtain

$$
\begin{aligned}
\left|a_{2} a_{4}-a_{3}^{2}\right| & =\left|\frac{1}{12}\left(-p_{1}^{4}+4 p_{1} p_{3}-3 p_{2}^{2}\right)\right| \\
& \leq \frac{1}{12}\left(\left|p_{1}\right|^{4}+4\left|p_{1}\right|\left|p_{3}\right|+3\left|p_{2}\right|^{2}\right) \\
& \leq \frac{1}{12}\left(\left\{\frac{\left(\sqrt{k^{2}+4}-k\right) k}{2}\right\}+4 \frac{\left(\sqrt{k^{2}+4}-k\right) k}{2}\left(k^{3}+3 k\right)\left\{\frac{\sqrt{k^{2}+4}-k}{2}\right\}^{3}\right. \\
& +3\left(\left(k^{2}+2\right)^{2}\left\{\frac{\left(k-\sqrt{k^{2}+4}\right) k}{2}+1\right\}^{2}\right) \\
& =\frac{2 k^{4}+6 k^{2}+3}{3}\left\{\frac{\sqrt{k^{2}+4}-k}{2}\right\}^{4} .
\end{aligned}
$$

Conjecture. If $f(z)=z+a_{2} z^{2}+\ldots$ belongs to $\mathcal{S} \mathcal{L}^{k}$, then

$$
\left|a_{2} a_{4}-a_{3}^{2}\right| \leq\left\{\frac{\sqrt{k^{2}+4}-k}{2}\right\}^{4} .
$$

The bound is sharp.

Theorem 9. If $f(z)=z+a_{2} z^{2}+\ldots$ belongs to $\mathcal{K} \mathcal{S} \mathcal{L}^{k}$, then

$$
\left|a_{2} a_{4}-a_{3}^{2}\right| \leq \frac{3 k^{4}+9 k^{2}+4}{36}\left\{\frac{\sqrt{k^{2}+4}-k}{2}\right\}^{4} .
$$


H. Özlem Güney, J. Sokół, S. İlhan - Second Hankel determinant problem ...

Proof. For given $f \in \mathcal{K} \mathcal{S} \mathcal{L}^{k}$, define $p(z)=1+p_{1} z+p_{2}^{2} z^{2}+\cdots$, by

$$
1+\frac{z f^{\prime \prime}(z)}{f^{\prime}(z)}=p(z)=1+p_{1} z+p_{2}^{2} z^{2}+\cdots,
$$

where $p \prec \tilde{p}$ in $\mathbb{U}$. Hence

$1+\frac{z f^{\prime \prime}(z)}{f^{\prime}(z)}=1+2 a_{2} z+\left(6 a_{3}-4 a_{2}^{2}\right) z^{2}+\left(12 a_{4}-18 a_{2} a_{3}+8 a_{2}^{3}\right) z^{3}+\cdots=1+p_{1} z+p_{2}^{2} z^{2}+\cdots$

and

$$
a_{2}=\frac{p_{1}}{2}, \quad a_{3}=\frac{p_{1}^{2}+p_{2}}{6}, \quad a_{4}=\frac{p_{1}^{3}+3 p_{1} p_{2}+2 p_{3}}{24} .
$$

Therefore, using Theorem (6) and Theorem (7), we obtain

$$
\left|a_{2} a_{4}-a_{3}^{2}\right| \leq \frac{3 k^{4}+9 k^{2}+4}{36}\left\{\frac{\sqrt{k^{2}+4}-k}{2}\right\}^{4} .
$$

Especially, if we take $k=1$ in Theorem (8) and Theorem (9), we obtain the results of Sokól et al. in [18]as follows:

Corollary 10. If $f(z)=z+a_{2} z^{2}+\ldots$ belongs to $\mathcal{S} \mathcal{L}$, then

$$
\left|a_{2} a_{4}-a_{3}^{2}\right| \leq \frac{11}{3}\left\{\frac{\sqrt{5}-1}{2}\right\}^{4} .
$$

Corollary 11. If $f(z)=z+a_{2} z^{2}+\ldots$ belongs to $\mathcal{K} \mathcal{S} \mathcal{L}$, then

$$
\left|a_{2} a_{4}-a_{3}^{2}\right| \leq \frac{4}{9}\left\{\frac{\sqrt{5}-1}{2}\right\}^{4}
$$

Acknowledgement This research has been supported with grant number FEN.17.026 by DUBAP (Dicle University Coordination Committee of Scientific Research Projects). The authors would like to thank DUBAP for their supporting and the referees for the helpful suggestions.

\section{REFERENCES}

[1] Dziok J., Raina R.K., Sokół J., Certain results for a class of convex functions related to a shell-like curve connected with Fibonacci numbers, Comp. Math. with Applications, (61)2011, 2605-2613. 
H. Özlem Güney, J. Sokół, S. İlhan - Second Hankel determinant problem ...

[2] Dziok J., Raina R. K., Sokół J., On $\alpha$-convex functions related to a shelllike curve connected with Fibonacci numbers, Appl. Math. Computation, 218(2011), 996-1002.

[3] Ehrenborg, R., The Hankel determinant of exponential polynomials, Amer. Math. Monthly, 107(2000): 557-560.

[4] M. Fekete, G. Szegö, Eine Bemerung über ungerade schlichte Functionen, J. Lond. Math. Soc. 8(1933) 85-89.

[5] Janteng A., Halim S. , Darus M., Coefficient inequality for a function whose derivative has a positive real part, J. Inequal. Pure Appl. Math., 7(2)(2006), Article 50 .

[6] Janteng A. , Halim S. , Darus M. , Hankel determinant for starlike and convex functions, Int. J. Math. Anal., 1(13)(2007) 619-625.

[7] Keogh, F. R. and Merkes, E. P., A coefficient inequality for certain classes of analytic functions, Proc. Amer. Math. Soc., 20(1969) 8-12.

[8] Layman, J. W., The Hankel transform and some of its properties, J. Integer Sequences, 4(2001): 1-11.

[9] R. J. Libera, E. J. Złotkiewicz, Coefficient bounds for the inverse of a function with derivative in $\mathcal{P}$, Proc. Amer. Math. Soc., 87(2)(1983) 251-257.

[10] Noonan, J. W. and Thomas, D. K., On the second Hankel determinant of areally mean p-valent functions, Trans. Amer. Math. Soc., 223(2)(1976) 337-346.

[11] Noor, K. I., Hankel determinant problem for the class of functions with bounded boundary rotation, Rev. Roum. Math. Pures Appl., 28(8)(1983) 731-739.

[12] Özgür N. Y. and Sokół J., On starlike functions connected with $k$-Fibonacci numbers, Bull. Malaysian Math. Sci. Soc., 38(1)(2015) 249-258.

[13] Pommerenke Ch., Univalent Functions, in: Studia Mathematica Mathematische Lehrbucher, Vanderhoeck and Ruprecht, Göttingen, 1975.

[14] Raina R. K. and Sokół J., Fekete-Szegö problem for some starlike functions related to shell-like curves, Math. Slovaca, 66(2016) 135-140.

[15] J. Sokół, On starlike functions connected with Fibonacci numbers, Folia Scient. Univ. Tech. Resoviensis 175(23)(1999), 111-116.

[16] J. Sokół, Remarks on shell-like functions, Folia Scient. Univ. Tech. Resoviensis 181(24)(2000), 111-115.

[17] Sokół J., Raina R. K. and Özgür N. Y., Applications of k-Fibonacci numbers for the starlike analytic functions, Hacettepe J. Math. and Statistic, 44(1)(2015) $121-127$.

[18] Sokół J., İlhan S. and Güney H.Ö., Second Hankel determinant problem for several classes of analytic functions related to shell-like curves connected with Fibonacci numbers, TWMS Journal of Pure and Applied Mathematics, accepted. 
H. Özlem Güney, J. Sokól, S. İlhan — Second Hankel determinant problem ...

Hatun Özlem Güney

Department of Mathematics, Faculty of Science, University of Dicle,

Diyarbakır, Turkey

email: ozlemg@dicle.edu.tr

Janusz Sokół

Faculty of Mathematics and Natural Sciences

University of Rzeszów,

Rzeszów, Poland

email: jsokol@ur.edu.pl

Sedat Ilhan

Department of Mathematics, Faculty of Science, University of Dicle,

Diyarbakır, Turkey

email: sedati@dicle.edu.tr 